\title{
Impact of composition and morphology on the optical properties of Si-NC/P3HT thin films processed from solution
}

\author{
F.D. Rodrigues $\cdot$ M. Cunha $\cdot$ L. Hilliou $\cdot$ L. Rino $\cdot$ \\ M.R. Correia - T. Busani - G. Bernardo · H. Wiggers • \\ S.A. Filonovich $\cdot$ R.N. Pereira
}

Received: 14 July 2012 / Accepted: 31 December 2012

(C) Springer-Verlag Berlin Heidelberg 2013

\begin{abstract}
Blends of Si nanocrystals (Si-NCs) and organic semiconductors are promising materials for new optical and electronic devices processed from solutions. Here, we study how the optical properties of composite films containing SiNCs and the organic semiconductor poly(3-hexylthiophene) (P3HT) are influenced by the composition and morphology resulting from different solution-processing parameters and different solvents used dichlorobenzene vs. chloroform). The optical spectra of the hybrid films are described using a simple phenomenological model, with which we can discern the contribution of each material in the films to the optical properties. From this analysis, we obtain quantitative information about the composition and morphology of the hybrid nanostructured films, which otherwise would be obtained from more demanding microscopy and
\end{abstract}

F.D. Rodrigues $\cdot$ L. Rino $\cdot$ M.R. Correia $\cdot$ R.N. Pereira $(\bowtie)$ Department of Physics and Institute for Nanostructures, Nanomodelling and Nanofabrication, University of Aveiro, 3810-193 Aveiro, Portugal

e-mail: rnpereira@ua.pt

M. Cunha $\cdot$ L. Hilliou $\cdot$ G. Bernardo

Institute for Polymers and Composites and Institute for Nanostructures, Nanomodelling and Nanofabrication, University of Minho, Campus de Azurém, 4800-058 Guimarães, Portugal

T. Busani $\cdot$ S.A. Filonovich

Cenimat, Institute for Nanostructures, Nanomodelling and Nanofabrication, Departamento de Ciência dos Materiais, Center of Excellence in Microelectronics Optoelectronics and Processes-Uninova, Universidade Nova de Lisboa, 2829-516

Caparica, Portugal

\section{H. Wiggers}

Institut für Verbrennung und Gasdynamik and Center for Nanointegration Duisburg-Essen, Universität Duisburg-Essen, 47048 Duisburg, Germany spectroscopy techniques. For the case of the Si-NC/P3HT blend, we find that in films deposited from dichlorobenzene solutions the Si-NCs contribute sizably to light absorption.

\section{Introduction}

Research on thin films containing semiconductor nanoparticles deposited from liquid dispersions of inorganic nanoparticles has extended rapidly in recent years for application in new optical and electronic devices [1-5]. Key advantages of film deposition from dispersions containing nanoparticles are the versatility and low cost provided by solution deposition methods, as well as the separation between the high temperature synthesis of the inorganic material and the material deposition, allowing deposition onto substrates not suitable for high temperature deposition. Freestanding silicon nanocrystals ( $\mathrm{Si}-\mathrm{NCs}$ ) are emerging as particularly interesting for such applications due to their unparalleled physical properties [6] and the ability of upscaling their synthesis to an industrial level. High quality Si-NCs can now be mass-produced in a cost-efficient and size-controlled manner using plasma-assisted decomposition of silane [7, 8]. Additionally, Si-NCs are made of a very abundant and environmentally inert element. In some of the envisaged applications, the Si-NCs are combined with an organic material. For instance, hybrid inorganic-organic light-emitting devices have been demonstrated using composites of Si-NCs and different polymers [9-11] and blends of Si-NCs and the hole-transporting polymer poly(3-hexylthiophene) (P3HT) have been used to demonstrate bulk-heterojunction solar cells $[12,13]$. Si-NCs are particularly promising for photovoltaics because their light absorption spectrum strongly 
overlaps with the solar spectrum, as demonstrated by the established success of crystalline silicon in the solar cell industry.

Despite the recent studies of optoelectronic devices based solution-processed films of Si-NCs and organic materials, detailed studies of the optical properties of such nanocomposite films are not yet reported in the literature. For instance, it is unclear which solvent is most appropriate to deposit Si-NC/organic layers with required properties. For example, in the case of films for photovoltaic applications, it is required that the Si-NCs are incorporated in the layers in such a way that their absorption of light is maximized, in order to take advantage of the remarkable optical properties of silicon for solar cells. A solvent well suited for the organic material is not necessarily convenient for the inorganic NCs and a good compromise has to be found. Both chloroform and dichlorobenzene have been used as solvent in the reported investigations of solution-processed hybrid Si-NC/organic devices [9-13]. The composition and morphology of hybrid films, which result from the deposition conditions such as solvent used, solution concentration, and deposition parameters, is detrimental for their use in applications. The morphology of such nanostructured materials may be obtained by high resolution microscopy techniques such as scanning electron microscopy (SEM) and transmission electron microscopy (TEM). However, these techniques are demanding and not always available. Since the optical properties of Si-NC/organic films depend strongly on their composition and morphology, the analysis of optical spectra may in principle be a faster alternate to obtain quantitative information about the composition and morphology.

In the present work, we study in detail the optical spectra of Si-NC/P3HT films deposited under different conditions using solutions of the Si-NC/P3HT mixture both in chloroform and in dichlorobenzene. The composition and morphology of the films is studied by SEM, micro-Raman spectroscopy, and X-ray diffraction (XRD). Using a simple phenomenological model, we describe the experimental spectra of the films and obtain quantitative information about their composition and morphology, which reasonably agrees with the results obtained from SEM, XRD, and microRaman spectroscopy. From this, we show that using optical measurements, one can quickly obtain information about the composition and morphology of NC/organic hybrid films, which otherwise are normally obtained from more demanding high-resolution microscopy and spectroscopy techniques. Furthermore, from our analysis of the optical spectra, we could discern the contribution of each material to the optical response of the films, and we show that for the case of the Si-NC/P3HT blend only in films deposited from dichlorobenzene solutions the Si-NCs contribute significantly to light absorption.

\section{Experimental details}

Freestanding Si-NCs were synthesized by microwave-induced decomposition of silane [7]. The Si-NCs have a spherical shape with a crystalline core and a surface $\mathrm{SiO}_{2}$ layer formed after exposure to air, with a thickness in the range of $1.4 \pm 0.2 \mathrm{~nm}$, as measured from high-resolution TEM imaging [14]. To remove the native oxide shell, the Si-NCs were etched in $\mathrm{HF}$ ( $10 \%$ in water) for 3 minutes and subsequently rinsed with ethanol. For this work, we synthesized Si-NCs with mean diameter of $48 \mathrm{~nm}$, determined by the BrunauerEmmet-Teller method [15]. Suspensions containing simultaneously P3HT (from American Dye Source, Inc.) and SiNCs in the weight proportion of 1:1 and total solid contents ranging from 1-4\% in weight were prepared in chloroform (CHL) and dichlorobenzene (DCB). To do this, P3HT solutions were first produced in each solvent by stirring at $60^{\circ} \mathrm{C}$ during 1 hour to achieve complete dissolution. Then Si-NCs were added and stirring of resulting suspensions was performed overnight under nitrogen atmosphere. Finally, the Si-NC/P3HT suspensions were sonicated to ensure a good dispersion of the Si-NCs (absence of aggregates to the naked eye). The substrates used in this work were glass slides covered with a thin film of poly(3,4-ethylenedioxythiophene) doped with poly-styrene sulphonic acid (PEDOT:PSS or PEDOT) deposited by means of spin-coating using a commercial solution (from Baytron P PV AI4083) and dried in air at $150{ }^{\circ} \mathrm{C}$ for 2 minutes, to ensure experimental conditions similar to those of previous studies of ligh-emitting devices and solar cells based on Si-NCs/polymer blends [9-13]. The resulting PEDOT:PSS films had a thickness of $45 \mathrm{~nm}$, as measured using a Dektak profilometer. Thin Si-NC/P3HT composite films were deposited on these substrates by means of spin-coating using the $\mathrm{Si}-\mathrm{NC} / \mathrm{P} 3 \mathrm{HT}$ dispersions described above. Spinning rates ranging from 500 to 6000 rotationsper-minute (RPM) and spinning times ranging from 10 to $120 \mathrm{~s}$ were tested. The resulting films were afterward annealed under vacuum at $150{ }^{\circ} \mathrm{C}$ for 20 minutes.

UV-Vis-NIR optical transmittance of all films was obtained using a Shimadzu UV-2100 spectrophotometer. The surface of the films was studied using high resolution SEM measured with a SU-70 Hitachi microscope, with a Schottky emission electron cannon using an operating voltage of $15 \mathrm{kV}$. Cross-section SEM images of the films were also obtained using a ZEISS Auriga cross beam instrument in focus ion beam (FIB) mode. Ga ions were used for the physical sputtering (milling) of the film and the images were recorded using the in-column SEM set up functioning at $5 \mathrm{KV}$ with a working distance of $5 \mathrm{~mm}$. In the case of films deposited from chloroform solutions, a thin protection layer of $\mathrm{Pt}$ was deposited onto the film surface prior to the milling. As shall be shown below (Fig. 7), this layer appears as a white region on the top of the films. Micro-Raman spectra of the 


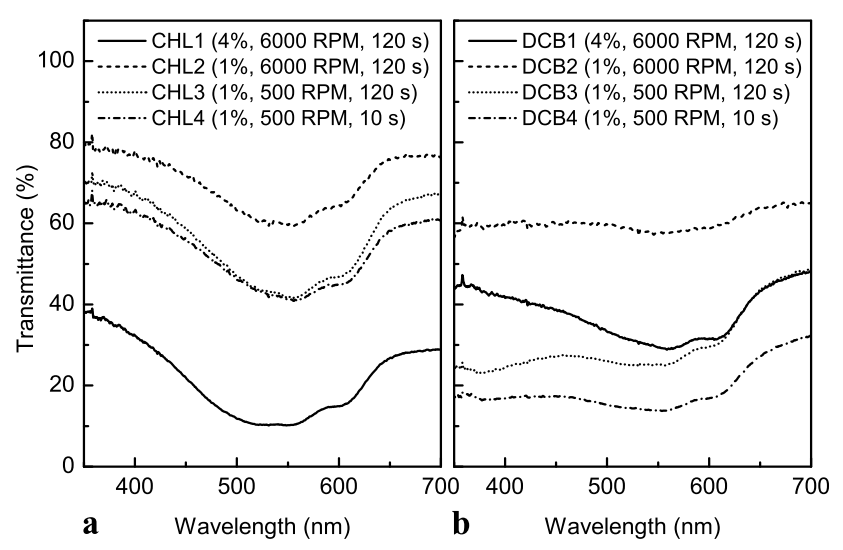

Fig. 1 Optical transmittance of Si-NC/P3HT films prepared from solutions in (a) chloroform and (b) dichlorobenzene using the indicated solution concentrations, rotation speed, and rotation time

films were recorded in back scattering configuration with a HR800 Jobin-Yvon spectrometer using a $532 \mathrm{~nm}$ laser excitation through a microscope system with a $100 \times$ amplification. The focused beam diameter was $\sim 1 \mu \mathrm{m}$ and the spectral resolution was $1 \mathrm{~cm}^{-1}$. XRD analysis of the films was performed under grazing-incidence using an $\mathrm{X}$ ray diffractometer (Bruker D8 Discover using $\mathrm{Cu} \mathrm{K} \alpha$ radiation, $\lambda=1.5406 \AA$ ) at a constant incidence angle of $1 \mathrm{de}$ gree. The scanning range was $2 \Theta=3$ to 40 degree with a step size of 0.04 degree.

\section{Experimental data and discussion}

Figures 1(a) and (b) represent the optical transmittance recorded for films prepared using solutions in chloroform and dichlorobenzene, respectively, under different deposition conditions. For example, CHL1 corresponds to a sample prepared using a $4 \%$ solution concentration and deposited using a speed of 6000 RPM during 120 seconds. Films CHL1 and CHL2 have been deposited with the same spin-coating parameters and from the same solvent, but from solutions with different concentrations (respectively 4 and $1 \%$ in weight). The lower concentration led to higher transmittance, which is due to the presence of less material in the film. When the same comparison is made for corresponding films but deposited from dichlorobenzene solutions [films DCB1 and DCB2 in Fig. 1(b)], we see that the difference in the transmittance, due to the difference in solution concentration, is attenuated. More important, for many of the films deposited from dichlorobenzene solution, a clear decrease of the transmittance with decreasing wavelength is observed. This effect is more pronounced for films DCB3 and DCB4, and is not observed for any of the films deposited from chloroform solutions, where the transmittances measured at $350 \mathrm{~nm}$ and $700 \mathrm{~nm}$ is about the same. The decrease of the transmittance with decreasing wavelength observed for films deposited from dichlorobenzene solutions occurs in the same range where a continuous increase of light absorption due to silicon is expected. Therefore, this effect could originate from light absorption in $\mathrm{Si}-\mathrm{NCs}$ present in the films. The influence of the deposition time on the transmittance of the films can be seen by, for example, comparing the samples DCB3 and DCB4, where an increase in rotation time led to higher transmittance. This effect is much weaker for films deposited from chloroform solution.

As stated above, some films deposited from dichlorobenzene solutions show a lowering in the transmittance spectrum with decreasing wavelength. In order to discover the origin of this effect, we investigated in more detail the structure and morphology of the film labelled DCB3, for which the effect is more pronounced. For comparison, we carried out a similar analysis of the film produced under the same conditions but using a chloroform solution, for which we did not observe a decrease in the transmittance with decreasing wavelength.

In Fig. 2, we compare SEM images of films deposited from chloroform [(a), (b)] and dichlorobenzene [(c), (d)]. As can be seen, the film processed from chloroform, labelled CHL3, contains large and well isolated agglomerates of Si-NCs. On the contrary, in the film processed from dichlorobenzene, labelled DCB3, the Si-NCs are more evenly distributed and the area of the film not covered with $\mathrm{Si}-\mathrm{NCs}$ is much smaller. In the SEM images of the chloroform processed film (CHL3), we can distinguish features with a dendrite shape [see Fig. 2(a)]. In order to evaluate structural/chemical differences between different regions of the film, we have carried out micro-Raman spectroscopy measurements. The capital letter labels in Fig. 2(a) indicate the regions where the micro-Raman spectra have been acquired in the case of the CHL3 film. The micro-Raman spectra are shown in Fig. 3(a). In the spectrum recorded in region A, which contains Si-NCs, a strong peak at $520 \mathrm{~cm}^{-1}$, typical of crystalline silicon [16], is observed. In addition, we observed lines at 1378 and $1446 \mathrm{~cm}^{-1}$ that originate from $\mathrm{C}-\mathrm{C}$ skeletal stretching and $\mathrm{C}=\mathrm{C}$ ring stretching modes of P3HT, respectively [17]. Peaks attributed to the PEDOT:PSS layer (indicated by the open black squares) are also observed at 575, 990, 1259, $1506 \mathrm{~cm}^{-1}$, which originate from oxyethilene ring deformation (first two referred peaks), asymmetric $\mathrm{C}_{\alpha}=\mathrm{C}_{\alpha^{\prime}}$ interring stretching, and asymmetric $\mathrm{C}=\mathrm{C}$ stretching, respectively [18, 19]. Additional peaks at 440 and $1569 \mathrm{~cm}^{-1}$ previously attributed to vibrational modes of PEDOT:PSS are also observed [20]. The spectra recorded on the regions not covered with $\mathrm{Si}$ NCs (spectra B and C) display peaks similar to those of region $\mathrm{A}$ with the exception of the crystalline silicon peak at $520 \mathrm{~cm}^{-1}$ [21], see spectra $B$ and $C$ in Fig. 3(a). The somewhat larger signal-to-noise ratio of these two spectra 
Fig. 2 SEM images of the surface of CHL3 [(a), (b)] and DCB3 [(c), (d)] films
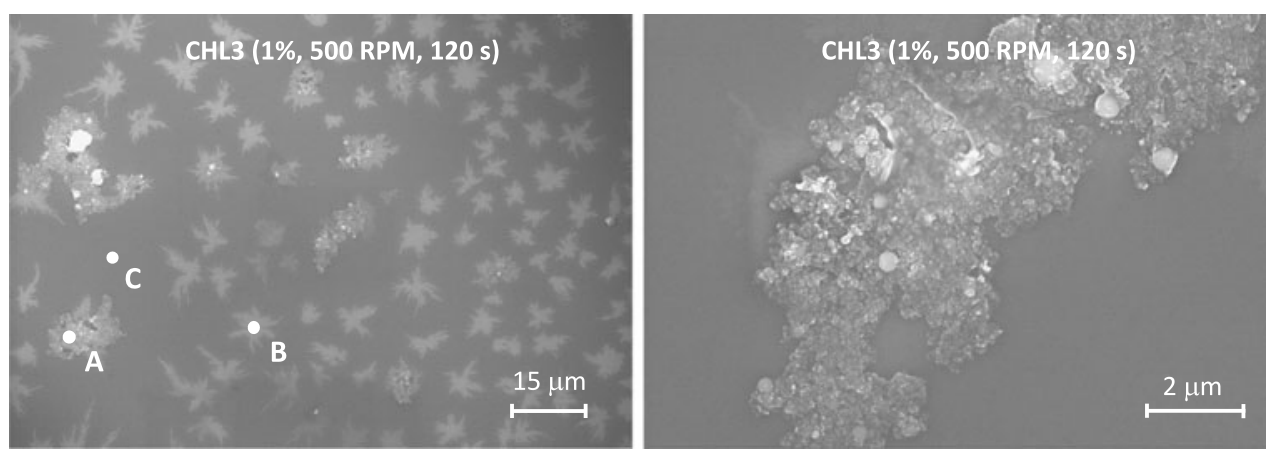

a

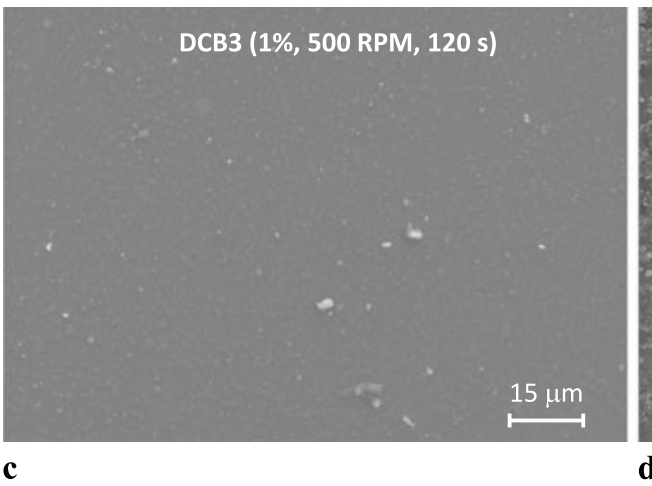

b

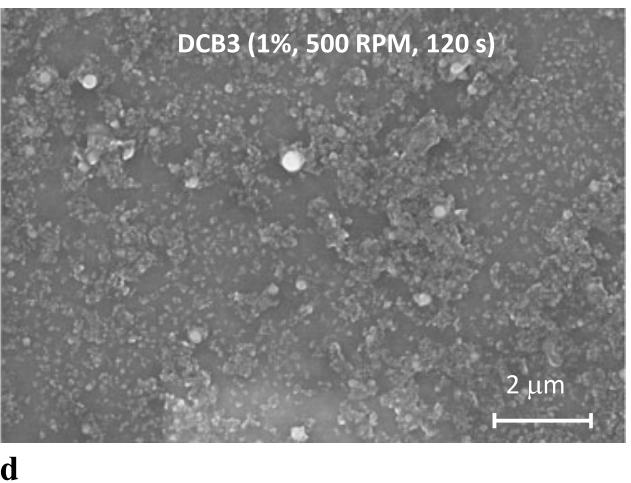

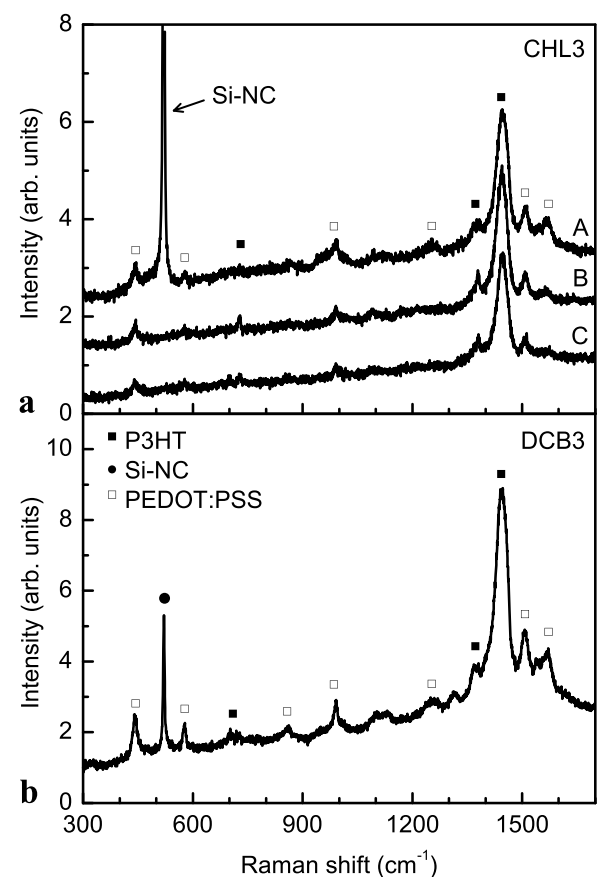

Fig. 3 (a) Micro-Raman spectra of a chloroform processed film (CHL3) recorded on the three different regions $\mathrm{A}, \mathrm{B}$, and $\mathrm{C}$ that are indicated in the SEM image of Fig. 2(a). (b) Micro-Raman spectrum of a dichlorobenzene processed film (DCB3)

enabled us to distinguish a small line at $726 \mathrm{~cm}^{-1}$ originating from the $\mathrm{C}-\mathrm{S}-\mathrm{C}$ ring deformation of $\mathrm{P} 3 \mathrm{HT}$ [17]. The relative intensity of peaks due to $\mathrm{P} 3 \mathrm{HT}$ and PEDOT:PSS is quite similar in the spectra recorded in the three regions A, $\mathrm{B}$, and $\mathrm{C}$. This indicates that the relative amount of the organic materials in these regions is similar. In particular, no significant chemical/structural difference in the $\mathrm{C}$ and $\mathrm{B}$ regions is distinguished. Therefore, we suggest that the dendrite structures can be due to a different contrast in the SEM image originating from charge accumulation in areas of the films with slightly different thickness.

In contrast to films deposited from chloroform solution, films deposited from dichlorobenzene do not exhibit large regions without Si-NCs, as can be seen in the SEM image of Fig. 2(c). This result is also confirmed by the micro-Raman studies, where we observe that Raman spectra recorded in random locations of the films surface are quite similar, and always display the silicon related peak. An example of such spectrum is shown in Fig. 3(b). Moreover, the relative intensity between the $529 \mathrm{~cm}^{-1}$ peak of Si-NCs and the $1446 \mathrm{~cm}^{-1}$ peak of P3HT is smaller in the Raman spectrum of the DCB3 film than in the spectrum obtained on region A of the CHL3 film. This indicates that in the region A of the CHL3 film the relative amount of Si-NCs with respect to $\mathrm{P} 3 \mathrm{HT}$ is larger than in the DCB3 film. We will return to this point below. We should also note that the somewhat smaller noise of spectra obtained from films produced from the dichlorobenzene solution, probably due to their smoother surface, enabled us to detect a peak at $859 \mathrm{~cm}^{-1}$ due to PEDOT:PSS [20] [see Fig. 3(b)], in addition to the peaks already found for chloroform processed films. 


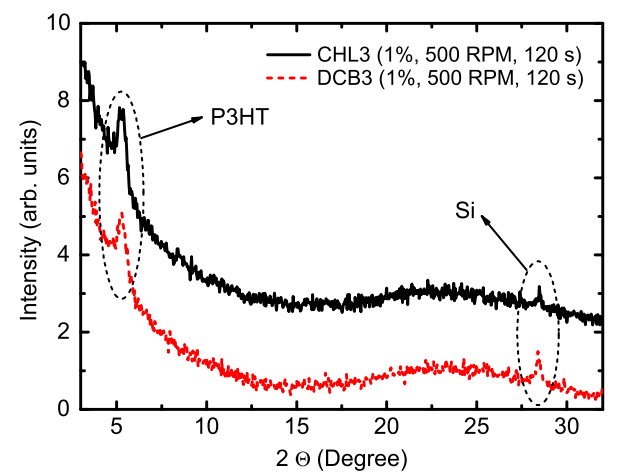

Fig. 4 X-ray diffractograms of CHL3 and DCB3 films

We have also studied the structure of the films by means of XRD. Figure 4 shows the X-ray diffractograms of two films. In the film processed from dichlorobenzene solution (DCB3), a peak can be found at $2 \Theta=5.3$ degrees corresponding to crystalline P3HT [22] and another peak can be found at $2 \Theta=28.5$ degrees, which originates from $\mathrm{Si}$ (111) [23]. Both peaks are also present in the XRD pattern of the chloroform processed films. An example is shown in the curve labelled CHL3 in Fig. 4. The peaks originating from P3HT in both films (DCB3 and CHL3) were fitted using Lorentzian curves with equal width, in order to determine their intensity. A similar approach was also applied for the Si (111) peak. These fittings enabled us to determine the P3HT/Si-NC XRD peak intensity ratio, whose values are 5.6 for the DCB3 film and 7.2 for the CHL3 film

Our combined micro-Raman and XRD data enable us to draw the following conclusions. The relative intensity of peaks related to P3HT and PEDOT:PSS is quite similar in the Raman spectra recorded in the CHL3 and DCB3 films. Taking into account that the amount of PEDOT:PSS in the two films is about the same, we can infer that the quantity of P3HT in these films should be similar. Moreover, if we consider this result and the ratio between the intensity of the XRD peaks from P3HT and Si-NCs in the same films (5.6 for DCB3 and 7.2 for CHL3), we conclude that the amount of Si-NCs in the two films should not deviate by more than $30 \%$. Thus, the amount of P3HT and SiNCs in the CHL3 and DCB3 films is somewhat similar and, therefore, the markedly different optical response of the two films should not originate from differences in their composition.

The optical spectra should depend strongly of the way the different materials are distributed in the films. To gain insight into the contribution of each material in the films to their optical properties, we carried out simulations of the optical spectra based on a simple model. From the information obtained from the SEM and micro-Raman analysis, we approximate the structure of the films to that illustrated in Fig. 5. Here, the film is composed of two types of multilayer systems, denoted (i) and (ii), with similar composi-

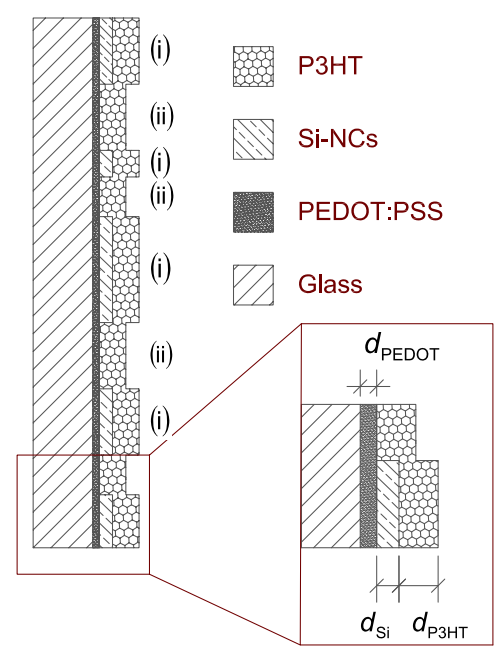

Fig. 5 Scheme of a cross-sectional view of the structure used in the model applied to describe UV-Vis-NIR spectra

tion with respect to the P3HT and PEDOT:PSS materials, but with region (i) containing Si-NCs. This model enabled us to take into account regions of the films that only contain the organic materials (PEDOT:PSS and P3HT) and regions that contain all three materials, which were revealed by the micro-Raman and SEM studies. Considering the structure shown in Fig. 5, the transmittance $T$ may be given by the expression:

$T=(1-R) \times \gamma_{\text {PEDOT }} \times \gamma_{\text {P3HT }} \times \gamma_{\mathrm{Si}} \times \gamma_{\mathrm{D}}$

where $R$ is the reflectance and the parameters $\gamma_{i}$ (with $i=$ PEDOT, P3HT, Si), representing the light absorption in each material, are given by

$\gamma_{\text {PEDOT }}(\lambda)=\exp \left[-\alpha_{\text {PEDOT }}(\lambda) \times d_{\text {PEDOT }}\right]$

$\gamma_{\mathrm{P} 3 \mathrm{HT}}(\lambda)=\exp \left[-\alpha_{\mathrm{P} 3 \mathrm{HT}}(\lambda) \times d_{\mathrm{P} 3 \mathrm{HT}}\right]$

$\gamma_{\mathrm{Si}}(\lambda)=\left[\beta \times \exp \left(-\alpha_{\mathrm{Si}}(\lambda) \times d_{\mathrm{Si}}\right)+(1-\beta)\right]$

$\alpha_{i}(\lambda)$ (with $i=$ PEDOT, P3HT, Si) are the absorption coefficients of the three materials reported in the literature [24-26]. $\gamma_{D}$ is a parameter accounting for light scattering. For the sake of simplicity, we consider here wavelengthindependent scattering given by the adjustable light diffusion parameter $\gamma_{D} \cdot{ }^{1}$ Moreover, $d_{i}$ (with $i=$ PEDOT, P3HT, $\mathrm{Si}$ ) represents the thickness of each material such as indicated in Fig. 5, where the thickness of the PEDOT:PSS layer

\footnotetext{
${ }^{1}$ Although light scattering may in general display a dependence on the wavelength $\lambda$, our analysis considers that in our type of films light scattering is mainly resulting from wavelength-independent light diffusion. Our approach has the merit of being very simple and of easy application, which is not the case of more complex scattering calculations based on Mie theory. Our approximation is justified by the fact that a reasonable description of our spectral data is attained, in combination with the fact that the morphological parameters obtained from our optical data analysis and the parameters obtained from the other techniques applied in the study agree reasonably well.
} 
Fig. 6 Ratio $T /(1-R)$ for the films CHL3 (a) and DCB3 (b) and respective curves resulting from the fittings, together with coefficients $\gamma_{i}$ ( $i=$ PEDOT, P3HT, Si, and D) obtained from the adjustments. Graphs (c) and (d) show the experimental transmittance $T$ and reflectance $R$ used to obtain the ratio $T /(1-R)$ for samples CHL3 and DCB3, respectively

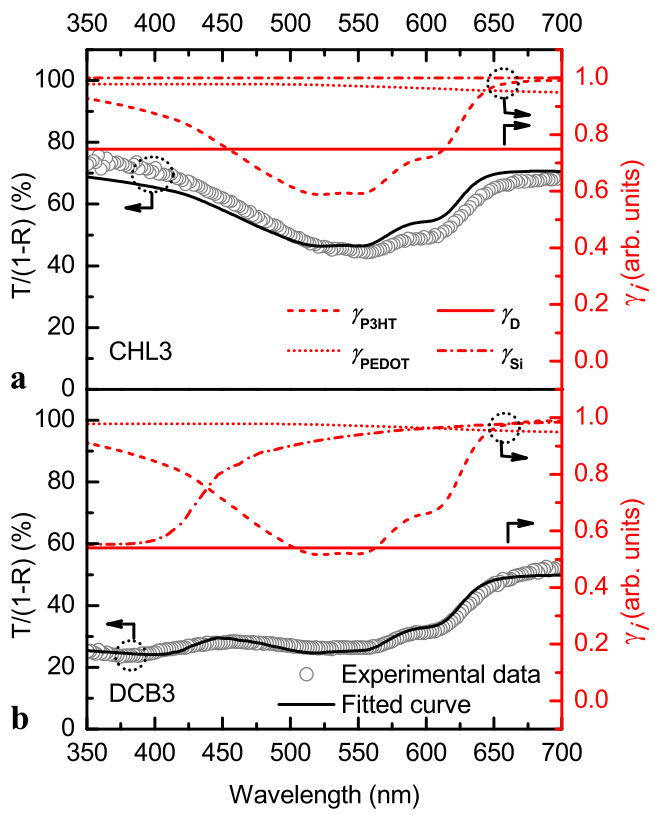

$\begin{array}{llllllll}350 & 400 & 450 & 500 & 550 & 600 & 650 & 700\end{array}$

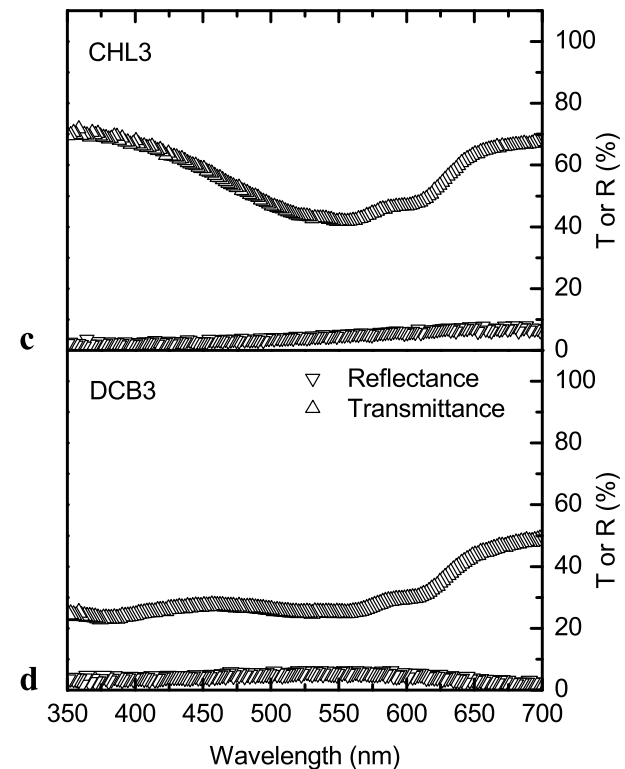

Table 1 Parameters and respective accuracies obtained from simulation of the optical spectra

\begin{tabular}{lllll}
\hline & $d_{\text {P3HT }}(\mathrm{nm})$ & $\beta$ & $d_{\mathrm{Si}}(\mathrm{nm})$ & $\gamma_{D}$ \\
\hline DCB1 $(4 \%, 6000 \mathrm{RPM}, 120 \mathrm{~s})$ & $26(5)$ & $0.0(0.1)$ & - & $0.47(0.07)$ \\
DCB2 $(1 \%, 6000 \mathrm{RPM}, 120 \mathrm{~s})$ & $8(2)$ & $0.14(0.04)$ & $63(24)$ & $0.64(0.09)$ \\
DCB3 $(1 \%, 500 \mathrm{RPM}, 120 \mathrm{~s})$ & $37(7)$ & $0.47(0.12)$ & $65(19)$ & $0.54(0.08)$ \\
DCB4 $(1 \%, 500 \mathrm{RPM}, 10 \mathrm{~s})$ & $47(9)$ & $0.39(0.10)$ & $58(22)$ & $0.34(0.05)$ \\
CHL1 $(4 \%, 6000 \mathrm{RPM}, 120 \mathrm{~s})$ & $60(12)$ & $0.0(0.1)$ & - & $0.37(0.05)$ \\
CHL2 $(1 \%, 6000 \mathrm{RPM}, 120 \mathrm{~s})$ & $13(3)$ & $0.0(0.1)$ & - & $0.85(0.12)$ \\
CHL3 $(1 \%, 500 \mathrm{RPM}, 120 \mathrm{~s})$ & $25(5)$ & $0.0(0.1)$ & - & $0.75(0.11)$ \\
CHL4 $(1 \%, 500 \mathrm{RPM}, 10 \mathrm{~s})$ & $21(5)$ & $0.0(0.1)$ & - & $0.68(0.10)$ \\
\hline
\end{tabular}

( $\left.d_{\text {PEDOT }}\right)$ corresponds to the value of $d_{\text {PEDOT }}=45 \mathrm{~nm}$ obtained from profilometry analysis, as described in Sect. 2. The parameter $\beta$ quantifies the area fraction of the film covered with Si-NCs with respect to the total area of the film.

Figures 6(a) and (b) show the ratio $T /(1-R)$ obtained from the experimental transmittance $T$ and reflectance $R$ recorded for the CHL3 and DCB3 films, respectively. The corresponding transmittance and reflectance spectra are shown in Figs. 6(c) and (d). From Eq. (1), it is seen that $T /(1-R)=\gamma_{\text {PEDOT }} \times \gamma_{\text {P3HT }} \times \gamma_{\mathrm{Si}} \times \gamma_{\mathrm{D}}$. We have fitted the experimental ratio $T /(1-R)$ with this expression and the results of this adjustment are shown in Figs. 6(a) and (b) for films CHL3 and DCB3, respectively. As can bee seen, a reasonable description of the experimental data is attained. For the DCB3 film, the parameters obtained from the fitting are $\beta=0.47 \pm 0.12, d_{\mathrm{Si}}=65 \pm 19 \mathrm{~nm}, d_{\mathrm{P} 3 \mathrm{HT}}=37 \pm 7 \mathrm{~nm}$, and $\gamma_{D}=0.54 \pm 0.08$ (see Table 1). For the CHL3 film, we obtained the values $d_{\mathrm{P} 3 \mathrm{HT}}=25 \pm 5 \mathrm{~nm}, \gamma_{\mathrm{D}}=0.73 \pm 0.11$, and $\beta=0.0-0.1$. We note that the recorded reflectance $R$ in all our films is quite low and, therefore, in our case $T /(1-R) \sim T$.

The parameters of the model determined from the adjustments of the spectra are in quite close agreement with the structure/morphology obtained from the SEM, microRaman, and XRD analyzes described above. Namely, the thickness of the P3HT layer ( $\left.d_{\mathrm{P} 3 \mathrm{HT}}\right)$ in the DCB3 and CHL3 films obtained from the fittings is similar $(\sim 30 \mathrm{~nm}$ for both films), which is in agreement with the information inferred above from Raman spectra described above. Moreover, the value of $\beta$ obtained for the DCB3 film $(0.47 \pm 0.12)$ is consistent with the film morphology understood from SEM imaging [see Fig. 2(d)], where we estimate that the area covered with Si-NCs in this film is about $60 \pm 10 \%$. In the case of the CHL3 film, the best fit occurs for $\beta=0.0(0.1)$. A small value for the parameter $\beta$ can also be consistently inferred from the SEM images [see Fig. 2(a)], from which we estimate a Si-NC coverage parameter $\beta \sim 0.06$. As can be seen from Eq. (4), a value of $\beta=0$ gives $\gamma_{\mathrm{Si}}(\lambda)=1$ for any value of $d_{\mathrm{Si}}$ and, therefore, the latter parameter cannot 


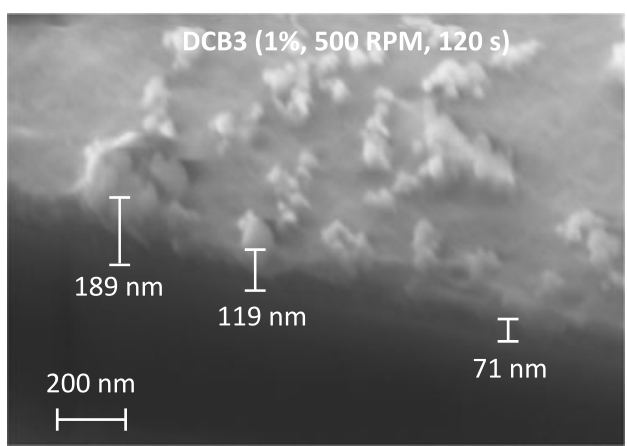

$\mathbf{a}$

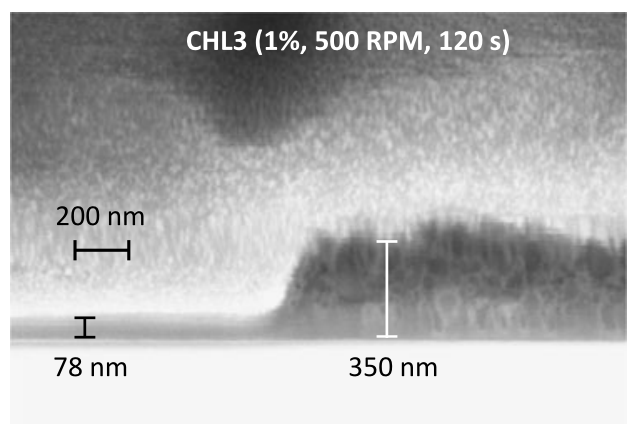

b

Fig. 7 SEM cross-section images of the (a) DCB3 and (b) CHL3 films. Both samples were tilted by 54 degrees with respect to the electron beam incidence axis. The shown images correspond to projections carried out to mimic a plane view situation. The white area evidenced in image (b) corresponds to the Pt protection layer deposited on top of the films, as described in Sect. 2

be reliably determined from the fitting. We should note that this does not mean that this film does not contain Si-NCs. In general, in films where $\beta$ is low $(\lesssim 0.1)$ the term $\gamma_{\mathrm{Si}}(\lambda)$ is very close to 1 , even if $d_{\mathrm{Si}}$ is relatively large. Thus, the Si-NCs may have a minute impact in the optical spectra of films that have a sizable amount of Si-NCs. We will discuss this further below.

To further verify the adequacy of our analysis of the optical spectra and the reliability of the quantitative information obtained from this analysis about the composition and morphology of the films, e.g., the thickness of each material in the films, we have carried out an additional study of the films cross-section by means of FIB/SEM. Examples of cross-section SEM images obtained for the DCB3 and CHL3 films are shown in Figs. 7(a) and (b), respectively. For the CHL3 film, the image shows both a region containing only the organic materials (left side) and a region where in addition also $\mathrm{Si}-\mathrm{NCs}$ are present (right side). Concerning the region containing only the organic materials, we observe a total thickness of the PEDOT:PSS and P3HT layers of $78 \mathrm{~nm}$. This value is remarkably close to that estimated from our analysis of the optical spectrum, i.e. $d_{\mathrm{PEDOT}}+d_{\mathrm{P} 3 \mathrm{HT}}=$ $70 \mathrm{~nm}$. The corresponding thickness obtained from fitting the DCB3 film spectrum is $d_{\mathrm{PEDOT}}+d_{\mathrm{P} 3 \mathrm{HT}}=82 \mathrm{~nm}$, which

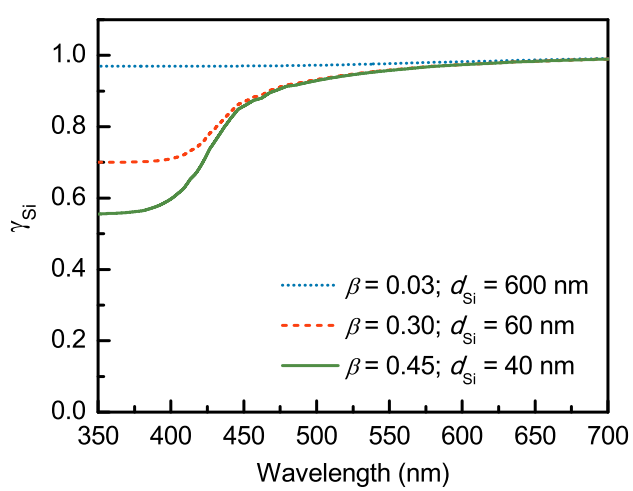

Fig. 8 Spectral dependence of the term $\gamma_{\text {Si }}$ given by Eq. (4) calculated for three different situations corresponding to films with equal amount of Si-NCs but different values of $\beta$ and $d_{\mathrm{Si}}$

is also close to the value of $71 \mathrm{~nm}$ observed from crosssection SEM imaging of regions uncovered with Si-NCs; see Fig. 7(a). In regions containing Si-NCs, we observe a total thickness in the range $150 \pm 40 \mathrm{~nm}$, which compares well with the corresponding value obtained from fitting the optical spectrum $d_{\text {PEDOT }}+d_{\mathrm{P} 3 \mathrm{HT}}+d_{\mathrm{Si}}=147 \mathrm{~nm}$. These comparisons confirm that our analysis of the optical spectra provides reliable information about the composition and morphology of the hybrid films.

The model parameters obtained from fitting the optical data of other films, shown in Fig. 1, are also given in Table 1 . As can be seen, the spectra adjustments indicate values of $\beta=0.0$ (0.1) for all films processed from chloroform solutions, independent of the deposition conditions. The small values of $\beta$ result in the fact that in films processed from chloroform solutions the $\mathrm{Si}-\mathrm{NC}$ do not contribute to a significant light absorption. From Eq. (4), we can see that when $\beta$ is small the term $\gamma_{\mathrm{Si}}$ is very close to 1 and, therefore, the Si-NCs have a minute influence on the transmission spectrum. This occurs even if the films contain a sizable amount of Si-NCs. This is, for instance, the case of the CHL3 film, where $\gamma_{\mathrm{Si}}$ approaches 1 due to a very small $\beta$, even though we know from the XRD and Raman analysis that these films contain a sizable amount of Si-NCs. Relatively large values of $\beta$ are obtained only for films processed from dichlorobenzene. The larger coverage of the films with Si-NCs, i.e. larger values of $\beta$, is obtained for dichlorobenzene processed films deposited at low rotation speed (500 RPM). Although the amount of Si$\mathrm{NCs}$ in these films can be comparable to that appearing in corresponding films processed from chloroform solution, in the dichlorobenzene processed films the Si-NCs are more evenly distributed on the films, i.e., $\beta$ is larger, which maximizes their impact on the optical transmission. To better exemplify this effect, we plot in Fig. 8 the term $\gamma_{\mathrm{Si}}$ for three situations corresponding to films with different values of $\beta$ and values of $d_{\mathrm{Si}}$ adjusted in order to maintain an equal amount 
of Si-NCs in all cases. As can be seen, for decreasing $\beta$, the term $\gamma_{\mathrm{Si}}$ approaches 1 , even though the $d_{\mathrm{Si}}$ is increased. This shows the importance of the distribution of $\mathrm{Si}-\mathrm{NCs}$ for the optical properties of the hybrid films. The better distribution of the Si-NCs in the dichlorobenzene processed films is most likely related to the higher level of dispersion of the NCs in this solvent prior deposition. The dispersion and thickness of the Si-NC subnetwork, quantified by the parameters $\beta$ and $d_{\mathrm{Si}}$, seem to be quite independent of the rotation time during deposition (compare, for example, the parameters obtained for the DCB3 and DCB4 films). Moreover, for both dichlorobenzene and chloroform processed films, the thickness of the P3HT sublayer $\left(d_{\mathrm{P} 3 \mathrm{HT}}\right)$ decreases when the concentration of the solution decreases (compare, for example, films CHL1 and CHL2) and is relatively insensitive to the spinning time during deposition (compare, for example, films CHL3 and CHL4).

\section{Conclusions}

The optical spectra of Si-NC/P3HT films deposited by spincoating under different conditions using chloroform and dichlorobenzene based solutions were studied. Using a simple model, we have described the experimental spectra and from such analysis we obtained quantitative information about the composition and morphology of the films. We have shown that these data agree reasonable well with those supplied by SEM, XRD, and micro-Raman spectroscopy experiments of the same films. From this, we demonstrate that using optical spectroscopy, one can quickly obtain quantitative information about the composition and morphology of hybrid films containing Si-NCs and organic materials, which otherwise would be obtained from more demanding high resolution microscopy and spectroscopy experiments. Furthermore, with the approach used in this work we could discern the contribution of each material to the optical properties of the films and showed that for the case of the SiNC/P3HT blend only in films deposited from dichlorobenzene solutions the Si-NCs contribute significantly to light absorption.

Acknowledgements This work was funded by FCT/I3N via the HybridSolar project, by the DFG via Teilprojekt B2 and by the European Union and the Ministry of Innovation, Science and Research of the German State of North Rhine-Westphalia in the framework of an Objective 2 Programme (European Regional Development Fund,
ERDF). Funding is also acknowledged from the Portuguese Foundation for Science and Technology (FCT) through the Projects Nos. PTDC/FIS/112885/2009 and PEst-C/CTM/LA0025/2011 and via the Strategic Project LA 25:2011-2012.

\section{References}

1. S.A. McDonald, G. Konstantatos, S. Zhang, P.W. Cyr, E.J.D. Klem, L. Levina, E.H. Sargent, Nat. Mater. 4, 138 (2005)

2. J.J. Urban, D.V. Talapin, E.V. Shevchenko, C.R. Kagan, C.B. Murray, Nat. Mater. 6, 115 (2007)

3. D.V. Talapin, C.B. Murray, Science 310, 86 (2005)

4. D. Yu, C. Wang, P. Guyot-Sionnest, Science 300, 1277 (2003)

5. B.A. Ridley, B. Nivi, J.M. Jacobson, Science 286, 746 (1999)

6. U. Kortshagen, J. Phys. D, Appl. Phys. 42, 113001 (2009)

7. A. Gupta, M.T. Swihart, H. Wiggers, Adv. Funct. Mater. 19, 696 (2009)

8. L. Mangolini, U. Kortshagen, Adv. Mater. 19, 2513 (2007)

9. R.K. Ligman, L. Mangolini, U.R. Kortshagen, S.A. Campbell, Appl. Phys. Lett. 90, 061116 (2007)

10. K.-Y. Cheng, R. Anthony, U.R. Kortshagen, R.J. Holmes, Nano Lett. 10, 1154 (2010)

11. K.-Y. Cheng, R. Anthony, U.R. Kortshagen, R.J. Holmes, Nano Lett. 11, 1952 (2011)

12. C.-Y. Liu, Z.C. Holman, U.R. Kortshagen, Adv. Funct. Mater. 20, $2157(2010)$

13. S. Niesar, W. Fabian, N. Petermann, D. Herrmann, E. Riedle, H. Wiggers, M.S. Brandt, M. Stutzmann, Green 1, 339 (2011)

14. R.N. Pereira, S. Niesar, W.B. You, A.F. da Cunha, N. Erhard, A.R. Stegner, H. Wiggers, M.-G. Willinger, M. Stutzmann, M.S. Brandt, J. Phys. Chem. C 115, 20120 (2011)

15. S. Brunauer, P.H. Emmett, E. Teller, J. Am. Chem. Soc. 60, 309 (1938)

16. R. Lechner, A.R. Stegner, R.N. Pereira, R. Dietmueller, M.S. Brandt, A. Ebbers, M. Trocha, H. Wiggers, M. Stutzmann, J. Appl. Phys. 104, 053701 (2008)

17. M. Baibarac, M. Lapkowski, A. Pron, S. Lefrant, I. Baltog, J. Raman Spectrosc. 29, 825 (1998)

18. S. Garreau, G. Louarn, J.P. Buisson, G. Froyer, S. Lefrant, Macromolecules 32, 6807 (1999)

19. G. Louarn, J.P. Buisson, S. Lefrant, D. Fichou, J. Phys. Chem. 99, 11399 (1995)

20. S. Sakamoto, M. Okumura, Z. Zhao, Y. Furukawa, Chem. Phys. Lett. 412, 395 (2005)

21. J.H. Parker, D.W. Feldman, M. Ashkin, Phys. Rev. 155, 712 (1967)

22. K. Sugiyama, T. Kojima, H. Fukuda, H. Yashiro, T. Matsuura, Y. Shimoyama, Thin Solid Films 516, 2691 (2008)

23. O. Ebil, R. Aparicio, S. Hazra, R.W. Birkmire, E. Sutter, Thin Solid Films 430, 120 (2003)

24. S. Günes, H. Neugebauer, N.S. Sariciftci, Chem. Rev. 107, 1324 (2007)

25. L. Pettersson, Org. Electron. 3, 143 (2002)

26. R. Lechner, Ph.D. thesis, Walter Schottky Institute, 2010 